\title{
De los inicios de la sinfonía a Beethoven: un paradigma de construcción europea
}

\author{
Emilio Fernando García Díaz
}

\author{
Recibido 10.04.2020 — Aceptado 5.05.2020
}

\section{Title / Titre / Titolo}

From the beginnings of Symphony to Beethoven: a paradigm of European construction

Dès les débuts de la symphonie jusqu’à Beethoven : un paradigme de la construction européenne

Dall'inizio della sinfonia a Beethoven:

un paradigma della costruzione europea

\section{Resumen / Abstract / Résumé / Riassunto}

Durante la primera mitad del siglo XVIII, la escena musical europea está protagonizada por diversos estilos instrumentales con un marcado acento nacional, resultado de la herencia del convulso siglo anterior, y que confluirán en la creación de un lenguaje instrumental que hará posible la materialización de la forma musical más importante dentro del género orquestal: la sinfonía. La obra de los grandes compositores que sentarán las bases del género sinfónico desde entonces, Haydn y Mozart principalmente, no hubiera sido la misma sin la síntesis integradora de estilos que se produce a lo largo del siglo. Distintos elementos extramusicales (filosóficos, políticos, sociales, etc.) inciden directamente en la búsqueda de un estilo europeo que armonizó lo mejor de cada tradición nacional para conformar un lenguaje que ha significado una de las producciones más importantes de nuestra historia musical. Culminado ese proceso y conocidas las consecuencias dramáticas de ese delirio racional que intentó iluminar a la sociedad europea, y que tan bien ilustró Francisco de Goya, Beethoven es capaz de sobreponerse al vacío que nos dejó el enorme desengaño de tan altos ideales, para volver a conjugar en su última sinfonía todos los elementos originales de aquella tradición dieciochesca y producir un universo sonoro que vuelve a recuperar algunos de los paradigmas más hermosos de nuestro devenir cultural. Más allá de las palabras de Schiller, este artículo intenta subrayar los elementos compositivos que se integran en la más célebre de las sinfonías beethovenianas como ejemplo inspirador para superación de las tentativas nacionalistas y los conflictos que hoy vuelven a surgir dentro de nuestro espacio europeo.

During the first half of the 18th century the European music scene is characterized by different instrumental styles with a clear national shape, result of the turbulent previous century, that will converge in the creation of the instrumental language that allowed the most important music form in the orchestral genre: symphony. The masterpieces that established the basis of the symphonic genre since then, Haydn and Mozart essentially, will not be the same without the synthesis of styles along the century. Non-musical elements (philosophical, politics, socials, etc.) affected directly the search of a European style that combined harmonically the best of each national tradition in order to build a language that constitute one of the most important productions in our musical history. Once reached the process and known the dramatic consequences of this rational dream that tried to enlighten the European society, so well-illustrated by Goya, Beethoven was able to overcome the emptiness created by the big disappointment of such high ideals, and was able to blend in his last symphony all the original elements of the $18^{\text {th }}$ century tradition and create a universe of sound that retakes some of the most beautiful model of our cultural development. Beyond Schiller' words, this article tries to underline the compositive elements integrated in Beethoven's most famous symphony as an inspiring example to get over nationalism and the conflicts that reappear today in our common European context.

Pendant la première moitié du XVIIIe siècle, la scène musicale européenne était dominée par divers styles instrumentaux à l'accent national marqué, résultat de l'héritage des bouleversements du siècle précédent, et qui convergeaient vers la création d'un langage instrumental qui allait permettre de matérialiser la forme musicale la plus importante au sein du genre orchestral : la symphonie. L'œuvre des grands compositeurs qui jetteront désormais les bases du genre symphonique, principalement Haydn et Mozart, n'aurait pas été la même sans la synthèse intégrative des styles produite tout au long du siècle. Différents éléments musicaux supplémentaires (philosophiques, politiques, sociaux, etc.) affectent directement la recherche d'un style européen qui harmonise le meilleur de chaque tradition nationale pour former un langage qui a signifié l'une des productions les plus importantes de notre histoire musicale. Une fois ce processus achevé et les conséquences dramatiques de ce délire rationnel qui a tenté d'éclairer la société européenne, et qui a été si bien illustré par Francisco de Goya, Beethoven est capable de surmonter le vide laissé par l'énorme déception d'idéaux aussi élevés, de réunir à nouveau dans sa dernière symphonie tous les éléments originaux de cette tradition du XVIIIe siècle et de produire un univers sonore qui reprend une fois de plus certains des plus beaux paradigmes de notre évolution culturelle. Au-delà des mots de Schiller, cet 
article tente de mettre en évidence les éléments de composition qui sont intégrés dans la plus célèbre des symphonies de Beethoven comme un exemple inspirant pour surmonter les tentatives nationalistes et les conflits qui surgissent aujourd'hui à nouveau au sein de notre espace européen.

Durante la prima metà del settecento la scena musicale europea è stata dominata da diversi stili strumentali con un marcato accento nazionale, frutto dell'eredità dei rivolgimenti del secolo precedente, confluiti nella creazione di un linguaggio strumentale che ha permesso di materializzare la forma musicale più importante all'interno del genere orchestrale: la sinfonia. L'opera dei grandi compositori che da allora in poi avrebbero posto le basi del genere sinfonico, soprattutto Haydn e Mozart, non sarebbe stata la stessa senza la sintesi integrativa degli stili prodotti nel corso del secolo. Diversi elementi extra musicali (filosofici, politici, sociali, ecc.) influiscono direttamente sulla ricerca di uno stile europeo che armonizza il meglio di ogni tradizione nazionale per plasmare un linguaggio che ha significato una delle produzioni più importanti della nostra storia musicale. Culminato il processo, con le drammatiche conseguenze del delirio razionale che cercò di illuminare la società europea, e che è stato ben illustrato da Francisco de Goya, Beethoven è in grado di superare il vuoto lasciato dall'enorme delusione di tali alti ideali, di combinare ancora una volta nella sua ultima sinfonia tutti gli elementi originali di quella tradizione settecentesca e di produrre un universo sonoro che recupera ancora una volta alcuni dei paradigmi più belli della nostra evoluzione culturale. $\mathrm{Al}$ di là delle parole di Schiller, questo articolo cerca di mettere in evidenza gli elementi compositivi che si integrano nella più famosa delle sinfonie beethoveniane come esempio ispiratore per superare i tentativi nazionalisti e i conflitti che oggi si ripresentano all'interno del nostro spazio europeo.

\section{Palabras clave / Keywords I Mots-clé / Parole chiave}

Tradición nacional, estilo europeo, síntesis, lenguaje instrumental sinfónico, Haydn, Mozart, Schiller, Beethoven, Goya.

National tradition, european style, synthesis, symphonic instrumental language, Haydn, Mozart, Schiller, Beethoven, Goya.

Tradition nationale, style européen, synthèse, langage instrumental symphonique, Haydn, Mozart, Schiller, Beethoven, Goya.

Tradizione nazionale, stile europeo, sintesi, linguaggio strumentale sinfonico, Haydn, Mozart, Schiller, Beethoven, Goya.

El artículo que aquí comienza no pretende conformar un análisis pormenorizado de los estilos que caracterizaron la escritura instrumental del XVIII ni de su posible contribución a la última sinfonía beethoveniana. Tan sólo, como simple intérprete que reconoce especial predilección por la obra de algunos autores que irán apareciendo en esta breve reflexión, intentaré hacer ac- cesible al lector no especializado algunos elementos estilísticos fundamentales para comprender la evolución del lenguaje sinfónico. Simplificaré, sin banalizar, algunos procedimientos técnicos que ya ocupan muchos artículos y tratados especializados con el objetivo de que el lector curioso que decida volver a escuchar algunas de las páginas de los autores referidos pueda disfrutar aún más de la audición activa. Si alcanzamos dicho objetivo a través de esta reflexión desde mi atril, bien merecerá la pena el esfuerzo y el tiempo dedicado a esta lectura.

\section{0, La herencia del siglo XVII}

\section{Aires italianos}

Para comenzar a dibujar los distintos estilos instrumentales predominantes a comienzos del XVIII, tenemos que hacer referencia al instrumento más perfecto: la voz. Esta aparente contradicción dejará de serlo si atendemos a la revolución que supuso en todos los ámbitos el nacimiento del género vocal que marcó el inicio del barroco: la Ópera.

En el intento de revivir la esencia del antiguo teatro clásico, el espíritu humanista del Renacimiento se concretó en una fórmula novedosa para la disposición de las voces que habían tejido el entramado polifónico imperante hasta entonces. La nueva melodía acompañada permitió un espacio de desarrollo a la voz principal, apoyada siempre en el bajo continuo, que se convirtió en la protagonista indiscutible de la obra. La influencia en la música instrumental de esta nueva forma de proceder es clara si atendemos a la producción instrumental del país donde nació, y desde el que se extendió a toda Europa, el género operístico. Sirvan de ejemplo las Sonatas Op. 5 para violín de Arcangelo Corelli' ${ }^{1}$.

Publicadas en Roma en 1700, junto a sus Conciertos Grossi, se convirtieron en modelos de referencia para compositores de todo el continente. En ellas encontra-

\footnotetext{
${ }^{1}$ A. Correlli, Sonatas para violín Op. 5: http://ks4.imslp.net/files/imglnks/usimg/ f/fb/IMSLP74741-PMLP28348-Corelli_-_12_Sonatas_Op_5.pdf.
} 
mos la mayoría de los elementos estilísticos y formales característicos de la música instrumental del barroco (forma bipartita apoyada en el esquema de transición armónica entre Tónica y Dominante en los movimientos de la suite, bajo continuo al estilo del trío sonata que sostiene el diseño motívico asociado a cada movimiento de danza, etc.), pero la melodía encomendada al violín, especialmente en la segunda parte de la obra, presenta una estructuración que, además de explorar los procedimientos propios del lenguaje instrumental del cual es heredero, dibuja un desarrollo melódico que denota una clara influencia del nuevo género vocal. La inspiración melódica, libre de los estrechos márgenes que permitía el entramado contrapuntístico, fue una de las características de la música italiana de la época, reconocida y admirada por todos los músicos del continente por su libertad de fraseo y la capacidad de transformación de los presupuestos motívicos que dan nombre a cada movimiento de la sonata instrumental. Tal y como declara Francesco Geminiani en su famoso tratado de violín (célebre por ser el primero destinado a la formación del violinista profesional): «El Arte de tocar el violín consiste en producir una sonoridad que pueda, de alguna manera, competir con voz humana más perfecta» (Geminiani, 1). Prueba de ello, es la evolución del arco italiano de la época, más largo y con menos curvatura convexa que su contemporáneo francés, apropiado para la imitación del Messa di voce del estilo vocal (procedimiento técnico de producción de sonido, especialmente indicado para las notas de mayor duración, que comienza de manera suave e incrementa gradualmente su intensidad al centro del arco para volver a decrecer hasta el final de la nota).

Si tomamos como ejemplo alguna de las sonatas de la Segunda Parte del Opus 5 de Corelli, (las Sonatas de la Primera Parte de la obra tienen, al contrario de lo que invitaría a pensar el desarrollo normal del corpus, un carácter más contrapuntístico y encierran mayor complejidad técnica de ejecución) el dibujo sonoro de la partitura nos permite perfilar una de las características fundamentales para el devenir lenguaje instrumental: la habitual imitación del motivo único que caracteriza cada movimiento de la suite está bien articulada por silencios periódicos que ordenan su desarrollo. Esta fragmentación regular de la línea melódica dota de gran claridad al discurso y recuerda, de alguna forma, la periodicidad natural de la pausa necesaria para la respiración vocal. Por supuesto, no quiero decir con ello que otros estilos instrumentales del periodo no utilicen el silencio como elemento estructural del desarrollo melódico. Sin embargo, sí que es significativa la periodicidad y regularidad en el caso de Corelli, sobre todo si lo comparamos con la densidad del discurso y la complejidad del fraseo del otro referente fundamental de la música instrumental de comienzos de siglo, Johann Sebastian Bach ${ }^{2}$.

Otro aspecto destacable en el lenguaje de Corelli, y que ha servido de tópico característico del estilo barroco italiano, es la repetición de pasajes, generalmente cadenciales, en forte y piano, el conocido recurso de eco (esta repetición no es común en la obra de Bach). Sin duda, este recurso estratégicamente dispuesto le sirve a Corelli para fijar la atención del oyente y prepararle ante un momento importante en la estructura de la obra, como puede ser un cambio de sección o antes de la cadencia final.

Los nuevos aires melódicos italianos se extendieron por toda Europa debido a la buena aceptación que tuvieron por el nuevo público burgués del XVIII, condicionando las distintas formas y grupos instrumentales. Es imposible entender la evolución de cualquier estilo musical sin atender a los distintos aspectos sociales, económicos, etc., de su contexto, que en este siglo están claramente marcados por el auge de la burguesía que desembocará en un reequilibrio de las relaciones sociales que marcaron el Antiguo Régimen. El nuevo público emergente demanda una música clara y sencilla, fácil de escuchar y disfrutar por el aficionado. Así, el compositor encuentra en este nuevo mercado una forma de ganar su sustento y liberarse de las condiciones impuestas desde las cortes reales o las capillas religiosas que habían ejercido como únicos patrones del mecenazgo artístico hasta entonces. Quizá no sólo por los

${ }^{2}$ J.S. Bach, Sonata en Sol M BWV 1021: http://ks4.imslp.net/files/imglnks/ usimg/f/f2/IMSLP534715-PMLP181438-D-LEb_Go._S._3,_Faszikel_1_ (BWV_1021).pdf 
condicionantes del mercado, sino por la comunión con los más elevados ideales humanistas ilustrados, el compositor va a encontrar un claro referente en la retórica del orador para lograr un modelo sonoro comprensible y capaz de satisfacer las expectativas del oyente. De esta manera, se comienzan a perfilar las estructuras motívicas con funciones de antecedente y consecuente articuladas regularmente en cadencias para conformar semifrases simétricas que desembocan en la frase cuadrada de ocho compases característica del Clasicismo. La repetición, la claridad y el orden se imponen con el denominado estilo galante, con el objetivo garantizar la compresibilidad del discurso musical.

Ejemplo paradigmático de la rápida difusión del nuevo estilo durante la primera mitad del siglo por toda Europa es la música de Giovanni Battista Pergolesi.

Para no olvidar el inicio de esta reflexión, la influencia de lo vocal en el género instrumental, volvemos a encontrar en la Ópera la causa, probable, de que la música instrumental del compositor napolitano tuviera tal repercusión en el continente en tan corto periodo de tiempo. La Serva Padrona, se convirtió pronto en el modelo del nuevo estilo operístico que arrasó en la escena lírica europea: la Ópera bufa. Este nuevo género, en contraste con la Ópera seria oficial, centró sus argumentos en temas cotidianos capaces de conectar con las inquietudes de un público más amplio, así como servir de plataforma a las nuevas ideas ilustradas y la fina crítica social.

El gran éxito de Pergolessi en la escena vocal le sirvió, sin duda, como carta de presentación para el resto de sus obras instrumentales que, aunque escasas, tuvieron un gran reconocimiento en todo Europa. De hecho, la fama y la admiración por la obra del napolitano transcendió al propio siglo: Igor Stravinski utiliza el tema de la Sonata en Sol Mayor para dos violines ${ }^{3}$ como motivo inicial de la obra que inaugura su periodo «clásico», el Ballet Pulcinella. La melodía que presenta el Violín I está perfectamente articulada en periodos simétricos con motivos repetidos que cadencian en Tónica

${ }_{3}^{3}$ G. B. Pergolessi, Trío no 1 en Sol menor: http://conquest.imslp.info/files/imglnks/usimg/4/41/IMSLP344272-SIBLEY1802.18092.4ac9-39087009052301score. pdf. o Dominante, mientras que el resto del trío acompaña sutilmente, sin interferir en la claridad de la línea principal. Igualmente, cuando en el compás ocho el Primer Violín cede el testigo melódico al Segundo Violín, su figuración se limita a notas largas que completan la armonía del acorde sin desviar la atención de la progresión melódica del segundo violín, que nos conduce hasta un periodo cadencial de dos compases repetidos en forte y piano para finalizar en la Dominante la primera sección del primer movimiento. Orden, simetría y claridad de líneas son los nuevos presupuestos que iluminarán buena parte del siglo.

No podemos dejar Italia sin hacer referencia a otros dos nombres fundamentales en la gestación del lenguaje sinfónico moderno.

Otro ilustre Giovanni Battista, Sammartini, en este caso, con más de setenta sinfonías que confieren al término el significado que desde entonces le relacionará con el género instrumental que todos conocemos. Utilizado de manera genérica durante el XVII para designar piezas instrumentales que sirven de introducción a obras de mayores dimensiones, sinfonía se utiliza como sinónimo de obertura en las óperas de ese siglo y se comienza a emancipar del género para convertirse, poco a poco, en una obra instrumental independiente y autónoma, aun conservando algunos elementos estructurales de su función primigenia. Así, la Sinfonía del milanés está, generalmente, dividida en tres movimientos con la estructura rápido-lento-rápido de la obertura italiana. El grupo instrumental al que va dirigido ya no responde a una amalgama diversa de instrumentos, fácilmente sustituibles por otros en caso de necesidad como sucedía anteriormente, sino que nos remite necesariamente a la familia de la cuerda frotada que sentará la base de la orquesta moderna ${ }^{4}$.

El otro gran nombre de la música instrumental italiana de la primera mitad del siglo XVIII es Antonio Vivaldi, quien recoge la herencia de Corelli para redirigirla, empujado por los nuevos aires de modernidad europea,

${ }^{4}$ G. B. Sammartini, Sinfonía en Sol Mayor, IGS 8: https://imslp.simssa.ca/files/ imglnks/usimg/4/4e/IMSLP433775-PMLP705102-EN324(2016)_-_Sammartini_GB_-_Sinfonia_G_major.pdf. 
y dejar una impronta que le concederá un lugar de privilegio en los escenarios y los gustos del público de todas las épocas. No sólo por el extraordinario desarrollo que aporta al concierto para instrumento, traduciendo al lenguaje instrumental el desarrollo e intercambio dramático de la escena entre el solo y tutti, sino por sus conciertos para orquesta y sinfonías de la última etapa de su obra en los que introduce técnicas orquestales que enriquecen las posibilidades sonoras del conjunto y que fueron tradicionalmente atribuidas, en exclusiva, a la invención alemana de la escuela de Manheim5.

\section{iVive la France!}

La música francesa de principios del siglo XVIII permanece ensimismada en la herencia de su propia tradición. La corte del Rey Sol fue el espejo en que se reflejaron todas las cortes europeas que quisieron alcanzar un estatus respetable, asumiendo con gusto los usos y protocolos afrancesados, al menos a comienzos de siglo.

La figura de otro Juan Bautista del siglo XVII, Jean Baptiste Llully, marcó el camino de la música de escena francesa y, asociada a ella, la música instrumental. Si la música italiana dio mayor espacio de desarrollo y transformación a la melodía, la ópera francesa aporta el contrapunto a la obertura italiana, lento-rápido-lento, y la inclusión de los ballets dentro de las óperas. Los aires de danza y el carácter cortés y solemne de las oberturas tienen una traducción en un motivo melódico corto, rítmico y preciso. La morfología del arco responde a estos presupuestos: frente al alargado arco italiano, el francés tiene una forma convexa más pronunciada, apropiada para los ritmos apuntillados, cortos y eléctricos en una especie de técnica puntillista con la que se perfila la melodía francesa. Quizá la mayor contribución de la música francesa al desarrollo melódico a comienzos del XVIII está en los aspectos cualitativos que adornan la melodía siempre bien articulada en periodos regulares:

5 A. Vivaldi, Concierto en Mi Mayor «La primavera»: http://imslp.eu/files/ imglnks/euimg/f/f2/IMSLP386586-PMLP126432-Vivaldi,_Antonio-Opere_ Ricordi_F_I_No_22_scan.pdf. los ornamentos con los que se enriquece la, aparentemente, sencilla línea melódica constituyen la seña de identidad de la música de compositores como Francois Couperin y el estilo Rococó del barroco tardío. En 1724, en el prefacio de su obra Les Goûts-réunis, on Nonveaux concerts, Couperin (1724) hace una honesta declaración de principios para que no haya lugar a la confusión entre las dos escuelas referidas:

La Música Italiana, teniendo el derecho de antigüedad sobre la nuestra, encontrará al final de este Volumen una gran Sonata a Trío titulada La Apoteosis de Corelli. Un aligera chispa de orgullo me ha llevado a presentarla en partitura. Si algún día mi musa se eleva por encima de ella misma, osaré a abordar también otro género, aquél del incomparable señor Llulli, del que sus obras deberían ser suficientes para inmortalizarlo. ${ }^{6}$

Además de las características propias del estilo nacional francés, claridad rítmica y formal, melodía fuertemente ceñida al acorde, gusto por lo pictórico, ornamentación, etc., en este breve «toun» por el estilo instrumental francés tenemos que resaltar un elemento estructural fundamental para la generación del lenguaje que aquí nos ocupa. Si Italia es la inspiración melódica, Francia es la gramática armónica que permitirá el desarrollo de un drama instrumental, aunque no adelantemos acontecimientos. La figura protagonista de esta esencial contribución es Jean-Philippe Rameau, un verdadero enciclopedista al servicio de la música. Su interés por la «ciencia» musical le llevó a formular la teoría de la armonía funcional que tendrá una influencia decisiva hasta el final de la Tonalidad (sistema de organización sonora que protagoniza nuestra tradición musical desde el Barroco hasta comienzos de siglo XX). Dotar a cada grado de la escala, y al acorde formado sobre él, de una función determinada, más allá de su sonoridad, fue la herramienta necesaria para poder crear un discurso instrumental independiente sin tener que recurrir a ningún otro elemento extramusical, llámese poema, libreto,

${ }^{6}$ F. Couperin, Les Goûts-réunis, ou Nouveaux concerts: http://ks4.imslp.net/files/imglnks/usimg/9/91/IMSLP29449-PMLP65940-couperin_gouts-reunis.pdf. Para el que quiera aventurarse en su interpretación se recomienda esta tabla de ornamentos del propio Couperin: https://images.app.goo.gl/dbV2PDKihHYHxvpm8; https://images.app.goo.gl/fWKG9A4m4yGvHYWq5. 
etc., y que cada sonido adquiera sentido dentro de un contexto musical autónomo. Contamos, pues, con un vocabulario y una gramática que permite la elaboración de piezas instrumentales cada vez más extensas y que tendrán buena acogida en los salones privados de la nobleza y la burguesía floreciente.

\section{El estilo estricto de la vieja peluca}

Mientras en Europa soplan los aires de modernidad que alumbran un nuevo estilo, un concienzudo artesano que intenta sacar lo mejor de sí mismo para cumplir con las obligaciones de su cargo y con su comunidad y, por encima de todo, ofrecer su trabajo a Dios, Johann Sebastian Bach, está escribiendo algunas de las mejores páginas del repertorio instrumental de nuestra historia, ajeno a los trending topic de la época y con una mirada puesta en la tradición, de la que se considera fiel y orgulloso heredero. Cultivó todos los géneros, excepto la Ópera, con maestría inigualable y reconocida por todos los músicos a partir de entonces, aunque se necesitó un poco de tiempo para entender el valor de su legado. Su obra pasó prácticamente desapercibida entre muchos de sus contemporáneos y fue ejemplo del denominado estilo «eclesiástico», «erudito» o «estricto», contrapuesto al «galante», siempre con ciertas connotaciones peyorativas por considerarse un género caduco y que no representaba los gustos y las necesidades de la nueva sociedad emergente. Poco pareció importarle a nuestro músico, que sí que contó con el respeto de la comunidad protestante alemana a quien dirigió su música.

La obra de J. S. Bach encarna la síntesis entre los estilos que se confrontaron a principios del siglo XVII desde la aparición de la Ópera: la monodia y la tradición polifónica renacentista. Los sistemas de afinación con los que se experimenta a lo largo del siglo, hasta desembocar en el temperamento igual o «bien temperado», permitirán el asentamiento de una gramática tonal que estructura todas las formas instrumentales contrapuntísticas en las que Bach es el maestro indiscutible. No sólo en la obra de órgano o teclado, sino incluso en las dedicadas a instrumentos como el cello o el violín solo, Bach despliega un entramado polifónico en el que exhibe todas las posibilidades del desarrollo motívico contrapuntístico (recordemos que cuando estas técnicas parecían ya en desuso, Bach escribe al final de su vida, entre 1749-50, El Arte de la Fuga, obra inacabada que resume su gran legado en este arte). Precisamente ahí es donde quisiera centrar mi reflexión sobre el significado de la obra de Bach para nuestro propósito: el desarrollo motívico.

Cualquier movimiento de una suite o sonata instrumental constituye un ensayo sobre el motivo que sirve de elemento generador de todo el movimiento. El motivo es el ADN donde se concentra todas posibilidades de desarrollo y transformación que se desplegarán en la pieza hasta conformar un universo autónomo y perfecto de relaciones musicales. No deja cabo suelto en su música, todo deriva de, o conduce a, los presupuestos originarios de la obra en un devenir constante, y sin pausa, hasta la cadencia final. El motivo es el concepto alrededor del cual se desarrollará el ensayo de su obra.

Aunque la grandeza del lenguaje de Bach podría ocupar toda esta humilde reflexión (creo que jamás me he aburrido de tocar una obra de Bach y cada vez que lo hago encuentro un nuevo motivo escondido y que me anima a seguir profundizando en ella), para no desviarnos de nuestros objetivos animo el lector interesado a escuchar y ojear el paisaje sonoro de una de las Sinfonías al estilo de la vieja peluca en sus años como director de música en la corte de Cöthen ${ }^{7}$, y que servirá para contrastar con el perfil del género que se dibujará unos años más tarde de la mano de sus hijos.

\section{Manheim}

Si queremos continuar con el modelo lingüístico, como lo hicieron los teóricos de la segunda mitad del XVIII

7 J.S. Bach, Sinfonía en Fa Mayor BWV 1046a: https://ks.imslp.net/files/imgl$\mathrm{nks} / \mathrm{usimg} / 3 / 34 /$ IMSLP565021-PMLP4549-Bach_-_Sinfonia_in_F_Major, BWV_1046a_-DVfM,_1956-.pdf 
(Koch, 1787; citado en Grout y Palisca, 1996: 449), para comprender el desarrollo del lenguaje instrumental durante esta época, una vez hemos hablado de la gramática armónica y la sintaxis de la frase melódica, debemos atender a la fonética musical. Este parámetro del sonido musical nos remite a la instrumentación y a la corte de Mannheim.

Aunque los aires ilustrados contribuyeron a la modernización de las comitivas palaciegas impulsando una intensa actividad cultural como bien universal, parece que el modelo a seguir fue aquél que simbolizó, como ningún otro, el Antiguo Régimen y el Absolutismo monárquico que ahora empieza a cuestionarse: la corte del Rey Sol y su famosa orquesta de los veinticuatro violines. Siguiendo este ejemplo, cualquier corte que quisiera ganarse el prestigio y el reconocimiento de sus iguales apostará por la formación de grupos instrumentales que contribuyan al disfrute y el regalo de su destacado séquito y el realce de los actos más solemnes. Así, cuando el príncipe elector Carlos III Felipe de Neoburgo traslada su corte de Heilderberg a Mannheim, conforma un grupo instrumental que juega un papel fundamental en la materialización del lenguaje sinfónico a partir de mediados del siglo XVIII. Para ello, contrata al joven virtuoso del violín Johann Stamitz, quien se encargará de organizar y dirigir la orquesta que maravillará a todos cuantos la escucharon:

No hay ninguna orquesta en el mundo que haya superado a la de Mannheim en concierto. Su forte es como un trueno, su crescendo como una catarata, su diminuendo como un arroyo de cristal que borbotea en la distancia, su piano un aliento de primavera. Los instrumentos de viento son empleados justo como deberían: sostienen y apoyan, o rellenan y animan, la tormenta de las cuerdas.

(Burney, 1775; citado en Rice, 2019, pp. 167-168).

Advierta el lector que el músico viajero inglés se centra en las dinámicas del grupo y en otra cualidad del sonido a la que no hemos hecho referencia y que será fundamental en el lenguaje sinfónico: el timbre. Stamitz es quien dibuja las secciones instrumentales y su función en la orquesta que protagoniza los primeros estadios del sinfonismo moderno. Introduce instrumentos en la plantilla orquestal como el clarinete y sienta las bases de la orquestación que utilizarán los grandes sinfonistas clásicos.

No será la orquestación el único aporte del músico bohemio a la formación de la sinfonía, sino que también incluirá en sus composiciones un Minueto y trío entre el segundo movimiento, lento, y el rápido final, ampliando el modelo italiano de tres movimientos (rápido-lento-rápido) y estableciendo la estructura de cuatro movimientos más común desde entonces.

\section{La construcción de un estilo europeo}

A mediados del siglo XVIII, las ideas ilustradas comienzan a calar profundamente en el ámbito musical y encontramos una clara tendencia a aunar los aportes de los distintos estilos nacionales con el objetivo de que esa especie de «síntesis superadora» (las comillas se justificarán más adelante) hegeliana recoja lo mejor de cada tradición para crear un estilo más universal y placentero. Sirvan de ejemplo las palabras del compositor y flautista Johann Joachim Quantz en su famoso tratado de 1752 (citado en Grout y Palisca, 443):

En un estilo que consiste, como el actual alemán, en la mezcla de estilos de gentes distintas, cada nación encuentra algo familiar e infaliblemente agradable. Considerando todo lo que ha sido analizado sobre las diferencias entre estilos, debemos inclinarnos a favor del puro estilo italiano sobre el francés. El primero ya no está tan sólidamente fundamentado como fue, habiendo degenerado en algo ostentoso y estrambótico, y el segundo ha permanecido demasiado simple. Todo el mundo coincidirá, por tanto, en que un estilo que aúna los mejores elementos de ambos será más universal y placentero. Para que una música sea aceptada y preferida por mucha gente, y no sólo por una sola tierra, provincia o nación particular, debe ser la mejor, siempre y cuando esté basada en el juicio sonoro y en una actitud saludable.

Note el lector que Quantz no hace mención a la tradición alemana, que obviamente nos remite a J. S. Bach. 
Parece que en el estilo que representa la «vieja peluca» no encuentra ningún elemento capaz de responder a los presupuestos de universalidad placentera que demanda el ansiado estilo europeo. No creo que al bueno de J.S. Bach le importase mucho, bastante tenía él con responder de la mejor manera posible a las obligaciones de su cargo y de su comunidad. De hecho, tampoco hizo nada, o poco, por dar a conocer su música. Entre las pocas salidas que hizo a alguna corte donde su música pudiera tener una proyección internacional se ha novelado frecuentemente la visita a la corte de Federico II de Prusia, corte en la que trabajaba su hijo Carl Philipp. El monarca prusiano, intérprete de reconocida valía y sabedor del arte del padre de su músico residente, aprovecha la visita del viejo Bach a su hijo para invitarle a su palacio y poder conocer su música en directo. En su cámara le pide tocar los numerosos instrumentos de tecla que posee y, según cuentan, le propone un tema para poder improvisar fugas y contrapuntos que eran su especialidad. Johann Sebastian así lo hace, pero no habiendo quedado satisfecho con sus improvisaciones, escribe tiempo más tarde su famosa Ofrenda musical.

Sin embargo, la neutralidad alemana que parece reflejar las palabras de Quantz en la construcción de un estilo musical europeo pronto acabará. Así, de la mano de autores como el propio Carl Philippe Emmanuel Bach y otros músicos que se reúnen en la corte de Federico II, se iniciará uno de los movimientos estéticos que promoverá el desarrollo de procedimientos compositivos armónicos que proporcionarán una base consistente para el drama instrumental sinfónico. Es el Empfindsamkeit o la Sensibilidad, cuyo ideal será provocar el continuo cambio en el estado emocional del oyente, a veces de manera imprevista, desembocará en la exploración de nuevos recursos armónicos, como la modulación o enlaces armónicos entre tonalidades más alejadas, necesarios para crear una trama instrumental y producir contrastes entre distintas secciones de un mismo movimiento o entre los temas asociados. Es decir, y esto es significativo para nuestros objetivos, el carácter de la música no permanecerá inalterado a lo largo de todo un movimiento, como ocurría anteriormente en las partes de la suite o en los movimientos de la sonata barroca, sino que la música fluctuará de un carácter a otro en el transcurso del mismo movimiento, sin solución de continuidad. La retórica musical ha conformado ya temas o ideas musicales bien definidas con una identidad suficientemente sólida para transformarse a lo largo del drama. Si bien el motivo es el concepto, el embrión que contiene la información genética suficiente para dar a luz una obra musical, el tema es una idea completa, compleja y compuesta de otras subordinadas que permiten desarrollar distintas escenas, colores o caracteres asociados y complementarios. En nuestra argumentación, asistimos, pues, al paso del desarrollo motívico al desarrollo temático. La nueva paleta de tonalidades que se abre a través de la modulación permitirá perfilar material melódico contrapuesto que interactúa a lo largo de la obra. Por tanto, el lenguaje instrumental en la segunda mitad del siglo XVIII cuenta ya con recursos compositivos necesarios para recrear un drama sonoro, sin necesidad de un libreto operístico o cualquier otra alusión externa al desarrollo musical, recursos tímbricos para caracterizar y perfilar la puesta en escena de cada personaje, o tema, y una estructura formal en varios movimientos, o actos teatrales, que permiten la elaboración de la obra compuesta y completa que es la sinfonía moderna.

Quizá, uno de los mejores ejemplos de la concreción sonora y formal de todo este proceso de desarrollo estilístico a lo largo de siglo XVIII es Carl Philipp Emanuel Bach. Como hemos visto, forma parte del selecto grupo de músicos e intelectuales que rodean a Federico II de Prusia en la corte berlinesa (junto a Quantz, Graun, etc.) y desde allí confabulan la verdadera revolución «sensible» de la que beberán los grandes nombres de la sinfonía moderna. De hecho, fue Carl Philipp quien aconsejó y sirvió de modelo a Haydn o Mozart y en su obra aparecen ya muchos de los elementos del nuevo lenguaje sinfónico ${ }^{8}$. Se observa, además, siguiendo el orden cronológico de sus sinfonías como se desarrollan los modelos barrocos y desaparece el bajo continuo, última reminiscencia del periodo preclásico, se amplía la

\footnotetext{
${ }^{3}$ C.P.E. Bach, Sinfonía en Re Mayor: http://ks4.imslp.info/files/imglnks/usimg/ d/dc/IMSLP79577-SIBLEY1802.12640.999d-39087009389877vol_3.pdf.
} 
plantilla orquestal y se perfila el drama instrumental a través de enlaces armónicos entre tonalidades más diversas que articulan secciones de un mismo movimiento y dan forma a la sinfónica clásica. Sin embargo, todavía nos queda citar a otro de los Bach, su hermano Johann Christian, para encontrar el último elemento característico del drama instrumental que desplegarán $\mathrm{Ha}$ ydn, Mozart y Beethoven en sus sinfonías: la interacción de dos temas en un mismo fragmento, forma sonata bitemática, frente a la forma sonata monotemática más característica de su hermano Carl Philipp o de Haydn en su primera época (Massin, 1987a, pp. 779, 785).

\section{La tradición que pervive}

Dejemos ya, de momento, a los grandes sinfonistas de la Primera Escuela de Viena escribir algunas de las grandes páginas de este género y culminar el desarrollo del estilo instrumental del XVIII. Volvamos a la perspectiva de nuestro atril, pero esta vez dejaremos la parte del Primer Violín, generalmente encargado de interpretar los temas y el material melódico más destacado de la obra, y cambiemos de asiento: el Segundo Violín. De Viena nos vamos a Boadilla del Monte para recalar en la música de uno de los compositores italianos más destacados dentro de la música instrumental de la segunda mitad del XVIII, Luigi Rodolfo Boccherini. En esta localidad, contratado en la corte de Don Luis Antonio Jaime de Borbón, escribirá su famoso minueto para quinteto de cuerdas. Sin embargo, por no desviarnos de nuestro género, centrémonos en una de sus maravillosas sinfonías de ese periodo,1771, Op. 12 n⿳3 en Do Mayor ${ }^{\circ}$, aunque las características que queremos señalar son las mismas que observamos en su música de cámara: la parte del Segundo Violín se limita generalmente a un acompañamiento rítmico-armónico de la melodía o a doblar, en ocasiones, al Primer Violín, al unísono o en terceras. Sin duda, se premia la voz principal y se elimina cualquier elemento que pueda desviar nuestra atención

9 L. Boccherini, Sinfonía no 3 en Do Mayor: https://ks.imslp.net/files/imglnks/ usimg/5/56/IMSLP250602-SIBLEY1802.18252.5283-39087009343379score.pdf. de ella. Esta característica es común a gran parte de la música instrumental del clasicismo temprano, tanto en sinfonías como en música de cámara. Quizá por ello, en una de las anécdotas que ilustran la niñez de Mozart, Schachtner, amigo y cronista de la familia, en una carta dirigida a Mariana Mozart en 1992, rememora como el niño de ocho años pide tocar a primera vista con su recién estrenado violincillo unos tríos del violinista y compositor Wentzl, junto a su padre, Leopold, el propio Wentzl y Schachtner. Ante la negación del padre, por no contar el niño con ninguna instrucción previa en el instrumento, el joven genio responde: «Pero, papá, para hacer la parte del segundo violín no es necesario haber aprendido» (Massin, 1987a: 58). La anécdota acaba con la mediación de Schachtner para que toque con él la parte del Segundo Violín, hasta que se da cuenta que el niño es capaz de leer perfectamente a primera vista la voz sin ninguna ayuda. Todo termina con lágrimas de admiración y elogios al joven prodigio.

Como intérprete, una sensación parecida he tenido en páginas de algunas sinfonías y cuartetos de Haydn, aunque no exactamente igual. Recordemos que «el padre de la sinfonía» es también quien otorga al cuarteto de cuerda el lugar de privilegio que la formación tendrá a lo largo de toda la historia dentro de la música de cámara, siendo él el primer maestro reconocido del género, especialmente a partir de 1772 con la publicación de su Op. 20. Con una parte de Primer Violín donde el compositor desarrolla toda su imaginación melódica y explota de manera genial los recursos técnicos del instrumento (cabe decir que incluso más que en los conciertos dedicados al violín), el Segundo Violín y la Viola despliegan un acompañamiento rítmico y melódico sutil desde el fondo de la escena, aunque siempre hay pasajes en los que el contrapunto contribuye a la tensión y el diálogo entre las voces (tres de los movimientos finales de su Op. 20 son fugas con dos, tres y cuatro sujetos). Esta interacción es más intensa cuando atacamos los cuartetos de Mozart y, sobretodo los de Beethoven, donde, especialmente a partir del periodo medio, las cuatro voces forman un complejo entramado polifónico entre los cuatro instrumentos. 
Unos años más tarde, en 1776, el joven Mozart escribe: «Vivimos en este mundo para aprender con aplicación y, por medio del intercambio de ideas, ilustrarnos unos a otros y con ello emprender la promoción de las ciencias y las bellas artes» (Anderson, 266).

No cabe duda de que las ideas «ilustradas» han calado profundamente en el sentir del gran genio salzburgués y los sinfonistas contemporáneos de la Primera Escuela de Viena. Sin ser éstos objeto de nuestra reflexión primera, ya que son precisamente ellos quienes encarnan la culminación del desarrollo del estilo instrumental del XVIII y su materialización sinfónica, sí que me gustaría incidir en un aspecto importante de las nobles palabras de Mozart: mientras que durante la primera mitad del siglo las tendencias más novedosas y renovadoras del lenguaje instrumental han venido de Italia y Francia, y así lo reconocía el propio Quantz, la culminación de ese proceso nos lleva a Austria y Alemania. ¿Se limitó el papel de estos países a «mezclar los estilos de gentes distintas» o añadieron una impronta de su tradición, aunque no aparezca declarada explícitamente en las palabras del flautista? Además de la riqueza armónica que aportaron movimientos como el Empfindsamkeit, en mi opinión, incorporaron una nueva lectura de su tradición contrapuntística que no se limita a la composición de fugas, especialmente en la música religiosa o en momentos puntuales de sus obras instrumentales. La nueva interpretación del contrapunto les permite abrir un espacio tridimensional para recrear en el desarrollo temporal lineal de la obra musical la interacción simultánea de los personajes de la escena dramática. Mientras que el estilo italiano parece seguir centrado en la traducción instrumental de la belleza del aria operística, el contrapunto permite reproducir la tensión coral de la escena teatral y enriquecer notablemente los recursos expresivos del lenguaje sinfónico.

Nadie duda de que Carl Philipp conociera la técnica contrapuntística a través de su padre, además de su formación como clavecinista, faceta en la que fue ampliamente reconocido tanto por su tratado como por sus composiciones para este instrumento. Sin embargo, no sólo él tuvo esa instrucción necesaria en la formación de cualquier músico: todos los grandes nombres de la primera gran escuela de sinfonistas contaron con una sólida formación contrapuntística. Si ojeamos la biografía del «padre de la sinfonía», Joseph Haydn, encontramos a un niño que a los ocho años entra como alumno en la escuela de capilla de la catedral de San Esteban de Viena donde, además de las clases en distintos instrumentos, practica y aprende los ejercicios de contrapunto del Gradus ad Parnassum de Johann Joseph Fux (1725), libro que, según su biógrafo, Griesinger (23),

seguía alabando en su vejez como un clásico del que había conservado un ejemplar muy desgastado. Haydn se esforzó incansablemente por comprender la teoría de Fux; estudió de forma práctica todo su método, hacía los ejercicios, los dejaba algunas semanas, volvía luego sobre ellos y los repasaba el tiempo necesario hasta que creía haberlos hecho satisfactoriamente.

En el caso de Mozart, el joven genio recibe su formación musical de su padre, Leopold Mozart, uno de los más metódicos pedagogos de su tiempo y famoso por su tratado de violín (1756), quien asumió la educación completa de su hijo sin que el pequeño Wolfgang pisara nunca una escuela. En las numerosas crónicas que deja escritas el orgulloso padre, y muchos otros admiradores, de los conciertos-espectáculo con los que viajó la familia Mozart por toda Europa desde que el pequeño prodigio tenía cinco años, destaca las dotes improvisadoras del joven Wolfgang, siendo capaz de arreglar en múltiples estilos cualquier melodía que le propusiese su distinguido público. Sin embargo, en Londres, con nueve años, un magistrado inglés, Daines Barrington, receloso del revuelo que ha causado la visita de los Mozart, le somete a un examen de sus conocimientos musicales que envió a la Real Sociedad de Londres. En dicho informe, da fe de la capacidad improvisadora del niño, capaz, entre otras habilidades, de continuar una fuga que el célebre Johann Christian Bach había comenzado, y «finalizarla de forma absolutamente magistral» (Massin, 1987a, p. 93). Así mismo, dado que el estilo religioso sigue muy presente en la isla, quizá por la gran influencia del Händel, Mozart ofrece al concluir su estancia 
en Londres un Motete al British Museum, para coro a cuatro voces en Sol menor «God is our refuge» $(\mathrm{K} .20)^{10}$, inspirado en el estilo eclesiástico antiguo con un verdadero tratamiento fugado de las voces.

\section{Beethoven: la síntesis superadora hegeliana}

La inspiración en la tradición contrapuntística es más evidente en Beethoven y, sobretodo, cuanto más avanza su obra. El genio de Bonn también tuvo una formación contrapuntística de la mano de Christian Gottlob Nefee, quien, a los doce años, le introdujo en el Clave bien temperado de J.S. Bach y las Sonatas de C.P.E. Bach. Un año más tarde el jovencísimo Louis es capaz de tocar «de corrido» la obra de J.S. Bach, según declaraciones del propio Neefe en el Magazin Musical de Cramer (Mas$\sin , 1987 b, 28)$.

Esta anécdota en la formación técnica del gran genio creador que revolucionó todos los modelos de sus predecesores, después de cultivarlos en su primer periodo, no es baladí: Beethoven encuentra en la tradición alemana una fuente inagotable de recursos para la regeneración del lenguaje instrumental que había nacido con unos presupuestos de universalidad ${ }^{11}$. Quizá, la contradicción entre lo nacional y lo universal, entre otras muchas contradicciones en diversos ámbitos de su propia vida, es la dialéctica donde nace la fuerza de su obra (los famosos cambios de la dedicatoria de la Heroica son sólo un ejemplo del conflicto interno). Sin embargo, no es este el espacio para tan elevadas reflexiones. Recuerdo que mi perspectiva es la de un humilde músico de atril, ahora rodeado de otros muchos colegas con los que en estos momentos estoy a punto de atacar el comienzo de la Quinta Sinfonía, un motivo que ha pasado a la historia de la música: «De este modo el destino llama a la puerta» (Massin, 1987b: 197). Sí, una vuelta declarada al

\footnotetext{
${ }_{10}$ W.A. Mozart, Motete en Sol menor K. 20: http://imslp.eu/files/imglnks/ euimg/0/0b/IMSLP421997-PMLP60341-nma_91_2_3.pdf.

1 En ningún momento he pretendido dudar de la formación contrapuntística de los músicos italianos o franceses, sólo incido en la diferente utilización de dichas técnicas en sus obras.
}

motivo, no al tema, que se anuncia sin complejos desde el comienzo de la obra y se desarrolla no sólo en el primer movimiento, sino en los otros tres que componen esta lucha del hombre contra su destino, pero tranquilos: parece que el cambio de Do menor a Do Mayor al comienzo del cuarto movimiento es señal indiscutible de que el hombre venció. Para celebrarlo, Beethoven amplía la paleta de la orquesta regular añadiendo un piccolo, contrafagot y trombones.

Esta mirada a la tradición local es bien patente en la obra de Beethoven con su gusto por los temas populares, y no sólo alemanes. Unos años antes de la Quinta Sinfonía, nuestro músico italiano nacionalizado, Boccherini, también utiliza elementos localistas en algunas de sus más famosas obras: Musica Notturna delle Strade di Madrid (1780) o la transcripción de 1796 de los quintetos de cuerda G. 270 y 341 para quinteto con guitarra, Quinteto $n^{0} 4$ en Re Mayor para guitarra ${ }^{12}$, con su famoso fandango que quedará ligado para siempre a la vida de otro sordo genial en la película de Carlos Saura, Goya en Burdeos. Estos elementos nacionales que enriquecen el lenguaje artístico serán interpretados como síntomas del primer Romanticismo que culminará en el XIX. Sin embargo, nosotros seguiremos nuestro concierto particular y la reflexión a partir de la grafía musical con otra gran fuga compuesta como movimiento final del cuarteto $n^{\circ} 13$ (¿guiño al Op. 20 de Haydn?). La dimensión colosal de esta fuga, forma contrapuntística por excelencia, y la fría reacción del público en su estreno lleva al editor, Artaria, a aconsejar, y rogar, su publicación por separado (Op. 133 ${ }^{13}$ ). Música absoluta rodeada de elementos extramusicales y literatura que la enriquece, pero música absoluta, porque sola se basta.

El contrapunto final a esa música absoluta y a estas líneas será la Novena Sinfonía: de nuevo un tema fugado en su último movimiento donde se conjugan todos los elementos que han ido apareciendo en nuestro relato: tema, motivo, armonía, contrapunto, instrumenta-

\footnotetext{
${ }^{12}$ L. Boccherini, Quinteto n ${ }^{\circ} 4$ en Re Mayor G 448: https://imslp.org/wiki/Guitar_Quintet_in_D_major\%2C_G.448_(Boccherini\%2C_Luigi).

13 L. Beethoven, Gran Fuga Op. 133: http://conquest.imslp.info/files/imglnks/ usimg/0/02/IMSLP51361-PMLP04536-Op.133.pdf.
} 
ción y voz para acabar, igual que se inició, este viaje por la música instrumental del XVIII. La oda de Schiller vuelve a iluminar las penumbras en las que las guerras napoleónicas sumieron a una Europa que soñó con valores de igualdad y fraternidad. Sólo la libertad del creador parece capaz de hacer revivir el sueño.

\section{Coda}

Los últimos años de Beethoven los protagonizan sus cuartetos de cuerda. «El monstruo de la música de cámara», según Schindler al respecto de la cavatina del Cuarteto $\mathrm{n}^{\circ} 13$, se había pintado unos años antes en los muros de la Quinta del Sordo, en el actual distrito de Latina. Beethoven sólo pone música a las pinturas que abrirán un nuevo campo de expresión tras la caída salvaje desde tan altos ideales. Sin embargo, el músico deja esbozada una Décima Sinfonía que le acompañará hasta sus últimos días. Sin duda, seguirá sonando en ella el motivo del hombre que se enfrenta a su destino y que sólo parece encontrar la paz en la naturaleza. No dejará de lado ningún rincón de individualidad nacional para enriquecer su lenguaje y encontrar en cada uno de ellos la universalidad y la fraternidad que nos haga superar las diferencias a las que el destino parece abocarnos. Esta sinfonía también será coral, aunque no sólo en ejecución, sino porque seamos nosotros quien debamos escribirla. No será fácil resolver las tensiones, no siempre armónicas, de los grandes retos a los que nos enfrentamos (escribo confinado y viendo desde la ventana como la naturaleza revive ahora que le damos tregua). El genio conocía bien el duro sacrifico de la creación, pero esta vez nosotros contamos con un camino ya perfilado en su novena. Quizá ha llegado el momento inexcusable para que la música adquiera verdadero significado y alcance la categoría de la disciplina lingüística tantas veces negada: la semántica.

\section{Bibliografía}

Anderson, Emily. The Letters of Mozart and His Family. Nueva York: Norton, 1985.

Geminiani, Francesco. The art of playing on the violin, 1751. Londres: Oxford University Press, 1952.

Grout, Donald Jay y Palisca, Claude. A History of Western Music. New York: Norton, 1996.

Griesinger, Georg August. Apuntes biográficos sobre Joseph Haydn. Madrid: Turner, 2012.

Massin, Jean y Brigitte. Wolfgang Amadeus Mozart. Madrid: Turner, 1987a.

Massin, Jean y Brigitte. Ludwig van Beethoven. Madrid: Turner, 1987b.

Mozart, Leopold. A Treatise on the Fundamental Principles of Violin Playing, trans. Editha Knocker (1948). Londres y Nueva York: Oxford University Press, 1756.

RicE, John A. La música en el siglo XVIII. Madrid: Akal, 2019.

\section{Índice de obras musicales}

BACH, Carl Philipp Emanuel, Sinfonía en Re Mayor: http://ks4.imslp.info/files/imglnks/usimg/d/dc/ IMSLP79577-SIBLEY1802.12640.999d-3908 7009389877vol_3.pdf.

BACH, Johann Sebastian, Sonata en Sol M BWV 1021: http://ks4.imslp.net/files/imglnks/usimg/f/f2/ IMSLP534715-PMLP181438-D-LEb_Go._S._3,_ Faszikel_1_(BWV_1021).pdf.

BACH, Johann Sebastian, Sinfonía en Fa Mayor BWV 1046a: https://ks.imslp.net/files/imglnks/usimg/3/34/ IMSLP565021-PMLP4549-Bach_-_Sinfonia_in_F_ Major,_BWV_1046a_-DVfM,_1956-.pdf.

BeEthoven, Ludwig van, Gran Fuga Op. 133: http:/ / conquest.imslp.info/files/imglnks/usimg/0/02/ IMSLP51361-PMLP04536-Op.133.pdf.

Boccherini, Luigi, Sinfonía no 3 en Do Mayor: https:/ / ks.imslp.net/files/imglnks/usimg/5/56/IM- 
SLP250602-SIBLEY1802.18252.5283-390870093 43379score.pdf.

Boccherini, Luigi, Quinteto no 4 en Re Mayor G 448: https://imslp.org/wiki/Guitar_Quintet_in_D_ major\%2C_G.448_(Boccherini\%2C_Luigi).

Correlli, Arcangelo, Sonatas para violín Op. 5: http:// ks4.imslp.net/files/imglnks/usimg/f/fb/IMSLP 74741-PMLP28348-Corelli_-_12_Sonatas_Op_5. pdf.

COUPERIN, François, Les Goûts-réunis, ou Nouveaux concerts: http://ks4.imslp.net/files/imglnks/usimg/9/91/ IMSLP29449-PMLP65940-couperin_gouts-reunis. pdf. Para el que quiera aventurarse en su interpretación se recomienda esta tabla de ornamentos del propio Couperin: https://images.app.goo.gl/dbV2P DKih HYHxvpm8, https://images.app.goo.gl/fW KG9A4m4yGv HYWq5.
MozArt, Wolfgang Amadeus, Motete en Sol menor K. 20: http:/ /imslp.eu/files/imglnks/euimg/0/0b/IMSL P421997-PMLP60341-nma_91_2_3.pdf.

Pergolessi, Giovanni Battista, Trío no 1 en Sol menor: http:// conquest.imslp.info/files/imglnks/usimg/ 4/41/IMSLP344272-SIBLEY1802.18092.4ac939087009052301score.pdf.

Sammartini, Giovanni Battista, Sinfonía en Sol Mayor, IGS 8: https://imslp.simssa.ca/files/imglnks/usimg/ 4/4e/IMSLP 433775 -PMLP 705102 -EN324 (2016)_-_Sammartini_GB_-_Sinfonia_G_major.pdf. VIVAldi, Antonio, Concierto en Mi Mayor «La primavera»: http://imslp.eu/files/imglnks/euimg/f/f2/ IMSLP386586-PMLP126432-Vivaldi,_AntonioOpere_Ricordi_F_I_No_22_scan.pdf. 
